\title{
Puntos de servicio en aseos públicos para minimizar y equilibrar los tiempos de espera de hombres y mujeres
}

\section{Service points in public toilets to minimize and balance the waiting times for women and men}

\author{
$\underline{\text { P. Grima }}^{(*)}$, Ll. Marco-Almagro ${ }^{(*)}$, X. Tort-Martorell $\left.{ }^{*}\right)$
}

RESUMEN

Se plantea el problema de las colas que se presentan habitualmente en los aseos de señoras en contraste con la fluidez con que se accede a los de caballeros, considerando que esta situación tiene solución ya que es sólo debida al mal dimensionado de los aseos. Tras describir y parametrizar los procesos de formación de colas en los aseos de señoras y de caballeros, se utiliza un programa de simulación para analizar en cada caso la influencia del número de puntos de servicio en los tiempos de espera. Finalmente, de acuerdo con los resultados obtenidos, se realizan unas recomendaciones para equilibrar los tiempos de espera, manteniéndolos en ambos casos por debajo de unos valores razonables.

Palabras clave: aseos públicos; aseos señoras; dimensionado aseos públicos; colas; tiempos de espera.

\section{ABSTRACT}

The article raises the issue of the queues that usually appear in the ladies toilets in contrast to the fluidity with which gentlemen can access theirs, and points that this situation is only due to bad dimensioning of the toilets and thus that the problem has an easy solution. After describing and parameterizing the queuing forming processes in ladies and gentlemen toilets, a simulation program is used to analyze in each case the influence of the number of service points in waiting times. Finally, the results obtained are used to give recommendations in order to balance the waiting times of ladies and gentlemen while keeping them below reasonable levels.

Keywords: public toilets; ladies toilets; dimensioning of public toilets; queues; waiting times.

(*) Departamento de Estadística e Investigación Operativa, Universitat Politècnica de Catalunya, Barcelona (España). Persona de contacto/Corresponding author: pere.grima@upc.edu (P. Grima) ORCID: http://orcid.org/oooo-0oo3-1470-1230 (P. Grima); http://orcid.org/oooo-ooo2-0440-1675 (Ll. Marco-Almagro); http://orcid.org/oooo-0003-1167-6703 (X. Tort-Martorell)

Cómo citar este artículo/Citation: Grima, P., Marco-Almagro, Ll., Tort-Martorell, X. (2017). Puntos de servicio en aseos públicos para minimizar y equilibrar los tiempos de espera de hombres y mujeres. Informes de la Construcción, 69(546): e191, doi: http://dx.doi. org/10.3989/ic.15.117.

Copyright: (C) 2017 CSIC. Licencia / License: Salvo indicación contraria, todos los contenidos de la edición electrónica de Informes de la Construcción se distribuyen bajo una licencia de uso y distribución Creative Commons Attribution License (CC BY) Spain 3.o. 


\section{INTRODUCCIÓN}

Cuando tras el incendio de 1994 se inauguró la reconstrucción del Gran Teatro del Liceo de Barcelona los comentarios fueron unánimes: se había conseguido el objetivo de combinar tradición con modernidad, había sido un éxito. El diario El Periódico (1) hacía un repaso a las opiniones de los expertos sobre la acústica («alabada por todos»), visibilidad («En general, se ha ganado en visibilidad...»), movilidad («amplio, cómodo...»). Todo muy bien, pero al llegar a los servicios se lee: «Los lavabos son modernos y luminosos, pero escasos en la planta de platea, a juzgar por las colas en los de señoras... Ellos entran y salen con fluidez, pero las señoras deben esperar a veces casi todo el entreacto.» Y ello a pesar de que -por supuesto- se cumplía con toda la normativa relativa al diseño de aseos en edificios públicos.

El problema de que las mujeres tengan que esperar más que los hombres para ir a los aseos no es un problema exclusivo del teatro del Liceo de Barcelona. Si se pregunta el porqué de este fenómeno, la respuesta suele ser que «las mujeres tardan más y es normal que tengan que esperar más». Pero dado que esto es así, y sobre ello no se puede actuar, hay que convenir que la verdadera causa de este problema es que los aseos están mal dimensionados.

Una solución evidente sería hacer más grandes los aseos de señoras, pero el espacio suele ser escaso y esta solución no siempre es posible. La solución «salomónica» de repartir la superficie disponible a partes iguales entre hombres y mujeres resulta muy desequilibrada en la práctica, tal como se pone de manifiesto más adelante. Una variante de este planteamiento sería poner los mismos puntos de servicio (váteres para mujeres y váteres más urinarios para hombres) tal como indica el «Reglamento General de Policía de Espectáculos Públicos y Actividades Recreativas» (2). De esta forma se destina más espacio a las mujeres, pero tampoco así se equilibran los tiempos de espera.

El presente artículo tiene como objetivo plantear esta problemática, poner de manifiesto que la normativa en vigor no la resuelve, y dar algunas recomendaciones para equilibrar los tiempos de espera de hombres y mujeres, manteniéndolos en ambos casos por debajo de unos valores razonables. Para ello se ha caracterizado el funcionamiento de los procesos de formación de colas que se siguen al ir al aseo en lugares públicos, se han identificado las variables que intervienen y, tras asignarles unos valores razonables, con la ayuda de un paquete de software para la simulación de procesos, se ha analizado el comportamiento de los tiempos de espera en función del número de puntos de servicio disponibles, tanto para hombres como para mujeres.

\section{ANTECEDENTES}

Es curioso como a pesar de lo universal de este problema -y de su importancia en la vida cotidiana- es poco tratado en la literatura técnica. Seguramente el libro que lo aborda con más detalle es el de Clara Greed (3), donde se presenta la evolución del diseño de los aseos públicos en el Reino Unido y se comenta como hace apenas 100 años el desequilibrio hombres/mujeres era todavía mayor, ya que en algunos casos simplemente no había aseos para mujeres. La descripción que realiza de los aseos en algunos teatros pone de manifiesto que el caso del Liceo de Barcelona no es único. Por ejem- plo, muestra los planos de los aseos del Glyndebourne Opera House y se observa que la superficie dedicada a señoras y caballeros es prácticamente la misma, pero mientras que en los primeros caben 3 váteres, en los segundos se incluyen 2 váteres y 4 urinarios (el doble de puntos de servicio para los hombres), lo cual inevitablemente implica un gran desequilibrio en los tiempos de espera. Comenta también que la situación está mejorando, ya que los aseos del Covent Garden Opera House han sido renovados, debido a las colas que se creaban en los de señoras, y que el Liverpool Philharmonic Hall también ha sido renovado con este mismo fin. A título orientativo sugiere que los aseos de señoras ocupen el doble de superficie que los de caballeros, y como referencia plantea un esquema general para los aseos de un lugar público concurrido colocando 3 váteres y 9 urinarios para los hombres y 18 váteres para las mujeres (lo que significa el 50\% más de puntos de servicio para las mujeres). Incluye también espacios para atender a bebés y para personas con mobilidad reducida, que son unisex.

La arquitecta Denise Scott Brown también trata el tema de los aseos de señoras en uno de sus artículos (4), lamentando que estén diseñados típicamente por hombres. Aboga por el uso de las matemáticas más que de la intuición y plantea temas como la necesidad de que haya colgadores para bolso o abrigos, pero no trata el tema de la distribución de puntos de servicio para hombres y mujeres.

Julia Edwards y Linda McKie (5) se plantean directamente el interrogante de «¿̇por qué las mujeres invariablemente tienen que hacer cola para entrar al baño en los lugares públicos, mientras que los hombres no?». Argumentan que las mujeres necesitan más baños y abogan -aunque sin ningún estudio cuantitativo que lo justifique- por situar el doble de puntos de servicio para mujeres que para hombres. También se han publicado artículos cortos como el de Jean Gaffin (6), donde se plantea la discriminación que representa el que haya escasez de aseos en lugares públicos, y en particular en los teatros, que obliga a las mujeres -y sólo a las mujeres- a hacer cola. También comenta que dado que las mujeres tardan el doble que los hombres, debería haber el doble de puntos de servicio para ellas.

Existen también libros sobre el diseño y la evolución de los cuartos de baño y los aparatos sanitarios, como los de Justo García y Eduardo de la Peña (7) (8), el de Ellen Lupton y J. Abbot Miller (9) o el de Alexander Kira (10). Este último realiza un minucioso análisis de la ergonomía de los diferentes aparatos -váteres, urinarios y lavabos-e incluso propone un diseño de urinarios para mujeres, pero ninguno de ellos trata del reparto de los puntos de servicio entre hombres y mujeres en los aseos públicos.

\section{NORMATIVAS}

En nuestro país tenemos el «Reglamento General de Policía de Espectáculos Públicos y Actividades Recreativas» antes citado (2), que en su artículo 12.1 indica que en edificios y locales destinados a espectáculos: «Se establecerán retretes, urinarios y lavabos en cada planta a razón de cuatro plazas de urinarios, dos inodoros y dos lavabos para caballeros y seis inodoros y dos lavabos para señoras, por cada 500 espectadores o fracción, reduciéndose aquellas cifras a la mitad en el caso de que el aforo de cada piso sea inferior a 300.» Es decir, se propone el mismo número de puntos de servicio para hom- 
bres y para mujeres, y como veremos más adelante ésta no es una buena solución.

Justo García Navarro y Eduardo de la Peña han publicado un Manual (11) que recoge las normas vigentes, tanto estatales como autonómicas, que se refieren a las instalaciones sanitarias. Respecto a los puntos de servicio en edificios de espectáculos públicos (apartado II.7), además del reglamento antes comentado, se incluyen los de ámbito autonómico para salones de juego, salas de bingo, espectáculos taurinos y campos de deporte. En todos los casos el número de puntos de servicio para mujeres es, como máximo, igual que para hombres.

La clasificación de normas de la International Organization for Standardization, ISO (12), incluye el apartado 91 que hace referencia a «Construction materials and Building», y dentro de éste existe el subapartado 91.140 .70 sobre «Sanitary Installations», pero que no está desarrollado. No existen normas UNE que hagan referencia al dimensionado de las zonas de hombres y mujeres en los aseos públicos, aunque sí existen documentos técnicos muy detallados sobre la accesibilidad de personas con discapacidad (13).

En el mundo anglosajón sí hemos encontrado normas que tratan con detalle este tema, aunque los criterios de aplicación no siempre están claros y las recomendaciones difieren bastante de unas a otras. Las British Standard, concretamente las BS 6465-1:2006 (14), dan recomendaciones para el dimensionado de los aseos e indican que deben realizarse cálculos separados para señoras y caballeros (apartado 8.4.3), que no debe haber cola en circunstancias normales y que una cola de más de dos minutos debe ser considerada inaceptable (apartado 8.4.1). A falta de estudios para cada caso concreto, proponen algunos valores a título orientativo:

- Cuando se tiene un tiempo limitado para ir al aseo (como en paradas de viajes largos) las visitas a los aseos pueden llegar a ser del $80 \%$ de los visitantes potenciales, mientras que si los aseos son accesibles en prácticamente cualquier momento (en un museo, por ejemplo) el porcentaje puede ser del $5 \%$ de las personas presentes.

- El tiempo que se tarda en usar el aseo se considera de 1,5 minutos para las mujeres y o,6 minutos para los hombres. Se puntualiza que si los hombres sólo disponen de váteres, su tiempo de uso también será de 1,5 minutos.

- En los aseos de caballeros debe haber 1 váter por cada 5 urinarios o fracción.

Sugieren que el número de puntos de servicio se calcule haciendo el producto del número de personas que acuden por unidad de tiempo -en el período de mayor afluencia- por el tiempo medio de uso, incluyendo un ejemplo con los valores que se reproducen en la Tabla 1.

De acuerdo con este criterio, si se considera que el número de visitas a los aseos es el mismo para ambos sexos, se deben poner 2,5 veces más de puntos de servicio para mujeres que para hombres (en la fórmula sólo cambia el tiempo de uso, que es 2,5 veces mayor en las mujeres). Por otra parte el resultado es muy sensible al período de tiempo considerado. $\mathrm{Si}$ este período se divide por dos, el resultado será el doble de puntos de servicio.

Existen también unas disposiciones técnicas sobre este tema que son más concretas y que se encuentran en el Código de Construcción Australiano (Building Code of Australia). En la parte referida a aseos y otras instalaciones (15) se incluyen tablas para cada tipo de local, donde se indica el número de váteres, urinarios y lavabos según el número de potenciales usuarios. La Tabla 2 reproduce los valores para váteres y urinarios en teatros y cines con un solo auditorio (página 305).

Naturalmente, el número de puntos de servicio es mayor para las mujeres, con un ratio que en general se encuentra entre 1,2 y 1,5 , aunque para algunos valores de potenciales usuarios (entre 101 y 110 de cada sexo) resulta el mismo en ambos casos. No se dice con qué criterios se han construido estas tablas ni cuáles son los tiempos de espera previstos.

Tabla 1. Ejemplo de cálculo de los puntos de servicio en los aseos de hombres/mujeres, incluido en las BS 6465-1:2006 (página 32).

\begin{tabular}{|l|c|c|}
\hline & Mujeres & Hombres \\
\hline Usuarios potenciales & 500 & 600 \\
\hline Porcentaje que utilizarán los servicios & $20 \%$ & $30 \%$ \\
\hline Tiempo de uso (en minutos) & 1,5 & 0,6 \\
\hline Período de tiempo considerado (hora punta, minutos) & 15 & 15 \\
\hline Puntos de servicio & $\frac{500 \times 0,20 \times 15}{15}=10$ & $\frac{600 \times 0,30 \times 0,6}{15}=8^{*}$ \\
\hline
\end{tabular}

* Siguiendo sus recomendaciones, se deberán colocar 6 urinarios y 2 váteres.

Tabla 2. Dotación de váteres y urinarios en un cine o teatro según el Código de Construcción Australiano.

\begin{tabular}{|c|c|c|c|c|}
\hline \multirow{2}{*}{ Grupo } & \multicolumn{2}{|c|}{ Váteres } & \multicolumn{2}{|c|}{ Urinarios } \\
\hline & Potenciales usuarios & Número & Potenciales usuarios & Número \\
\hline Hombres & $\begin{array}{c}1-50 \\
51-250 \\
251-500 \\
>500\end{array}$ & $\begin{array}{c} \\
1 \\
2 \\
\text { Añadir } 1 \text { por } 500 \\
\end{array}$ & $\begin{array}{c}1-50 \\
51-100 \\
>100\end{array}$ & $\begin{array}{c} \\
1 \\
\text { Añadir } 1 \text { por } 100\end{array}$ \\
\hline Mujeres & $\begin{array}{c}1-50 \\
51-110 \\
111-170 \\
171-230 \\
231-250 \\
>250\end{array}$ & $\begin{array}{c}\text { o } \\
3 \\
4 \\
5 \\
6 \\
\text { Añadir 1 por 80 }\end{array}$ & & \\
\hline
\end{tabular}




\section{CARACTERIZACIÓN DE LOS PROCESOS PARA SU SIMULACIÓN}

Se distingue entre la situación tipo «entreacto», donde la afluencia a los aseos se concentra en un corto período de tiempo, y la de «flujo continuo», en que las llegadas se producen de forma regular a lo largo del día (como en museos o monumentos). En ambas situaciones se sigue el mismo proceso, pero se tienen distintos valores para alguna de sus variables.

\subsection{Proceso en los aseos de señoras}

En el caso de las mujeres el diagrama de flujo del proceso es muy sencillo: van llegando y si no hay váteres libres hacen cola. Cuando un váter se queda vacío es ocupado por la primera persona de la cola (Figura 1).

Las variables y los valores considerados son:

- Afluencia: Es el número de personas que acuden a los aseos. Se han elegido unos valores que abarquen la mayoría de situaciones que se dan en la práctica. Para el modelo entreacto se han considerado afluencias de 20, 30, 40 y 50 personas, mientras que para el modelo de flujo continuo se han considerado unas llegadas de 30, 60, 90 y 120 personas por hora.

- Período de afluencia: En el modelo entreacto este valor afecta a los tiempos de espera, ya que si todas llegan en un tiempo muy reducido, las últimas deberán esperar más que si el período se alarga, de forma que cuando llegan las últimas las primeras ya han terminado. Considerar un período de afluencia igual al tiempo del entreacto, como parecen sugerir las British Standard, no es razonable, ya que nadie va al aseo justo antes de que vuelva a empezar el espectáculo. Se ha considerado razonable utilizar un período de afluencia de 5 minutos, de forma que las llegadas se reparten uniformemente en este período. En el caso del flujo continuo se ha considerado el período de una hora, que puede ser representativo de la afluencia del día en general, o de lo que ocurre en los períodos de máxima afluencia.
- Tiempos de uso: A falta de estudios detallados sobre este tema, hemos utilizado como valor de partida 1,5 minutos (90 segundos) que aparece en las British Standard (14). Se ha considerado que los valores se distribuyen de acuerdo con una distribución Normal (campana de Gauss) con el valor medio indicado y una desviación típica de 15 segundos [distribución que notamos como N(90; 15) con los valores en segundos]. Con este patrón de variabilidad la mayoría de las observaciones se presentan agrupadas en torno a la media, concretamente el 95\% se dan en el intervalo: media \pm 2 desviaciones típicas, y prácticamente ninguna (menos del $3 \%$ ) más allá de la media \pm 3 desviaciones típicas (16). Intentando reflejar lo que ocurre en la realidad, se considera que un cierto porcentaje de personas (10\% en el modelo entreacto y $15 \%$ en el de flujo continuo) va a estar en el váter un tiempo más largo de lo normal, y para éstas se supone una media de 2 minutos y una desviación típica de 20 segundos, es decir: N(120; 20) segundos. Además de utilizar estos valores para los tiempos de uso (que denominamos «hipótesis 1») se ha analizado también lo que ocurre cuando éstos son 15 segundos mayores («hipótesis 2»). Trabajar con dos hipótesis para los tiempos de uso también nos permitirá valorar la sensibilidad de los resultados ante esta variable.

- Número de WC: Se han considerado valores de entre 1 y 30 para el modelo entreacto y de entre 1 y 10 en el de flujo continuo.

Todos estos valores se hallan resumidos en la Tabla 3. Cabe destacar que a cada persona se le ha asignado una probabilidad de que su tiempo de uso pertenezca a la distribución de los tiempos largos y esta probabilidad es igual a la proporción establecida. De esta forma los tiempos largos se distribuyen al azar, aunque no siempre se reproducirá exactamente la proporción que se indica, tal como ocurre en la realidad.

\subsection{Proceso en los aseos de caballeros}

En el caso de los hombres se forma una cola general (si es necesaria) que avanza de la siguiente forma (Figura 2):
Afluencia:

Entreacto: 20, 30, 40, 50

Flujo continuo: $30,60,90,120$

Período de afluencia:

Entreacto: 5 minutos

Flujo continuo: por hora

\% de tiempo de uso largo:

Entreacto: $10 \%$

Flujo continuo: $15 \%$
Tiempo de uso, hipótesis 1:

Estándar: $\mathrm{N}(90 ; 15)$ segundos

Largo: N(120; 20) segundos

Tiempo de uso hipótesis 2:

Estándar: $\mathrm{N}(105 ; 15)$ segundos

Largo: N(135; 20) segundos

Número de váteres:

Entreacto: 1-30

Flujo continuo: 1-10

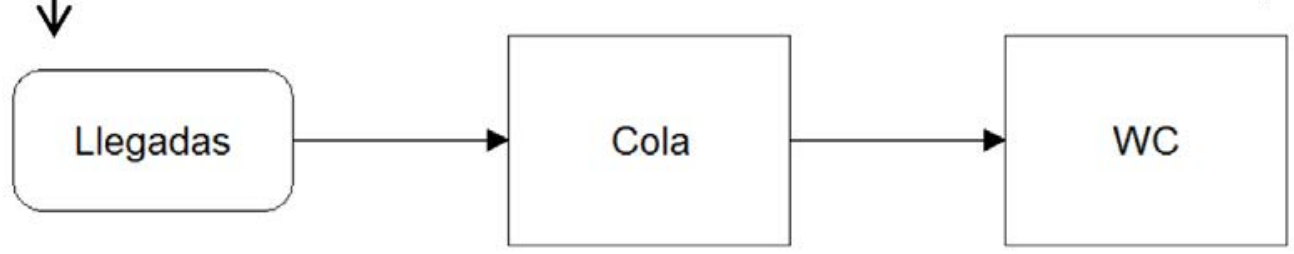

Figura 1. Esquema del proceso para el caso de las mujeres. 
- Si queda un urinario libre lo ocupa la primera persona de la cola si ése era su interés, o se aparta de la cola general esperando que quede un váter libre.
- Si queda un váter libre lo ocupa la primera persona de la cola tanto si su interés era de un váter como de un urinario.

Tabla 3. Valores de las variables que intervienen en la simulación del proceso en los aseos de señoras.

\begin{tabular}{|c|c|c|c|c|}
\hline & & & Entreacto & Flujo continuo \\
\hline \multirow{3}{*}{ Llegadas } & \multicolumn{2}{|c|}{ Número total de llegadas } & $20 ; 30 ; 40 ; 50$ & - \\
\hline & \multicolumn{2}{|c|}{ Ritmo de llegadas (por hora) } & - & $30 ; 60 ; 90 ; 120$ \\
\hline & \multicolumn{2}{|c|}{ Rango tiempo de llegada } & 5 minutos & por hora \\
\hline \multirow{5}{*}{$\begin{array}{l}\text { Tiempo de uso } \\
\text { (en segundos) }\end{array}$} & \multirow{2}{*}{ Hipótesis 1} & Estándar & \multicolumn{2}{|c|}{$\mathrm{N}(90 ; 15)$} \\
\hline & & Largo & \multicolumn{2}{|c|}{$\mathrm{N}(120 ; 20)$} \\
\hline & \multirow{2}{*}{ Hipótesis 2} & Estándar & \multicolumn{2}{|c|}{$\mathrm{N}(105 ; 15)$} \\
\hline & & Largo & \multicolumn{2}{|c|}{$\mathrm{N}(135 ; 20)$} \\
\hline & \multicolumn{2}{|c|}{ Proporción tiempo largo } & 0,10 & 0,15 \\
\hline Puntos de servicio & \multicolumn{2}{|c|}{ Número de WC } & $1-30$ & $1-10$ \\
\hline
\end{tabular}

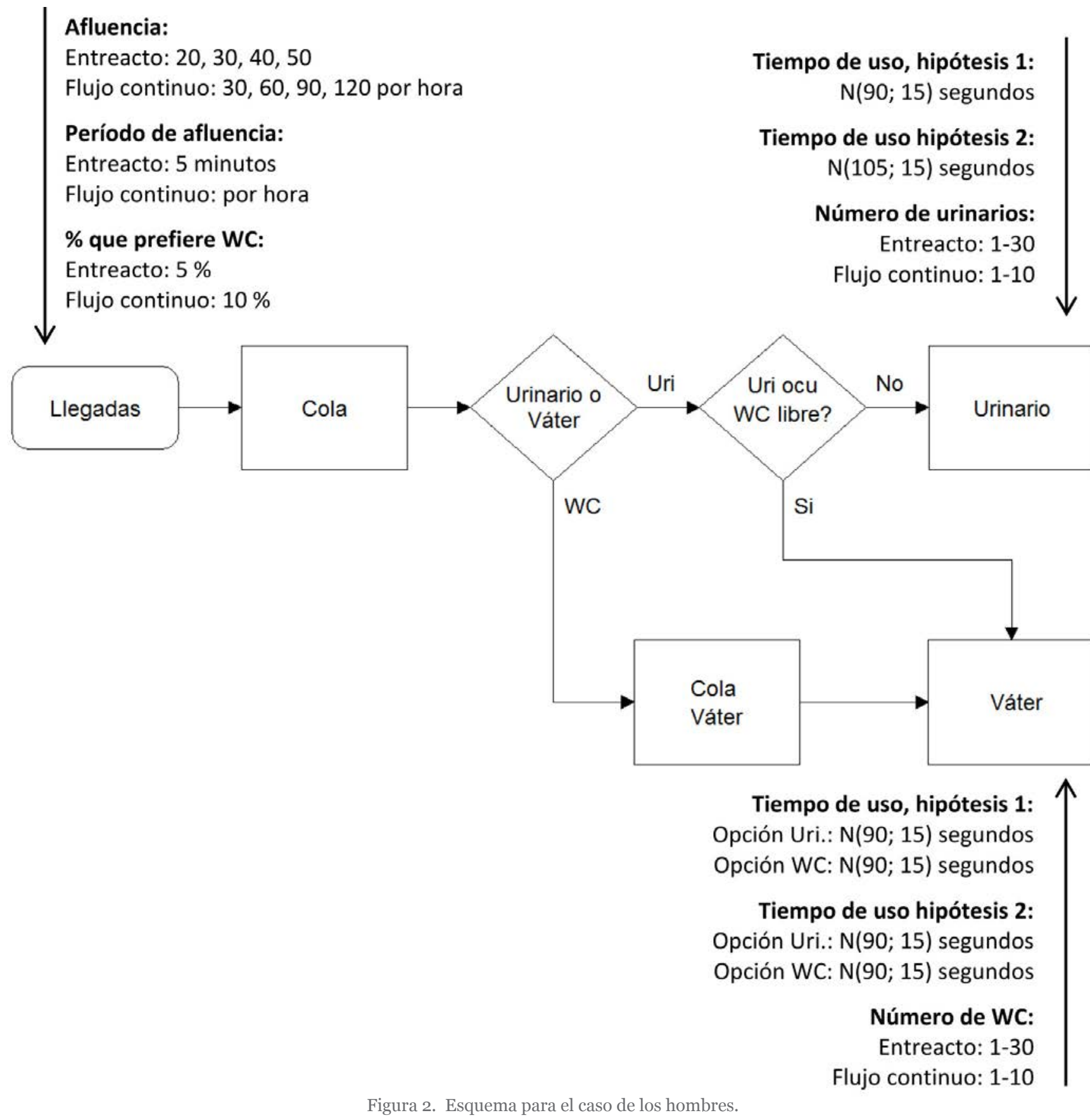

Figura 2. Esquema para el caso de los hombres. 
Las variables consideradas, así como sus valores asignados, son:

- Afluencia, período de afluencia: Para ambas variables se utilizan los mismos valores que para las mujeres tanto en el modelo entreacto como en el de flujo continuo.

- Porcentaje que prefieren váter: En el modelo entreacto se considera que el $10 \%$ de los hombres que se dirige a los aseos busca un váter. En el modelo de flujo continuo este porcentaje se ha considerado del $15 \%$. La asignación se ha realizado de la misma forma que para los tiempos largos en el caso de las mujeres.

- Tiempo de uso: Al igual que en el caso de las mujeres, se ha planteado una hipótesis (hipótesis 1) que utiliza los tiempos medios que figuran en las British Standard y otra (hipótesis 2) con valores algo superiores. Las distribucio- nes de estos tiempos son las que figuran en la Tabla 4. En este caso de los hombres, al hacerse ya la distinción entre los que prefieren urinario o váter, no se ha incluido la opción de «tiempo largo» como se ha hecho en el caso de las mujeres para no crear un modelo excesivamente prolijo. El tiempo medio de uso del WC bajo la hipótesis 2 se ha considerado igual a 115 segundos, que resulta un valor similar al de las mujeres, teniendo en cuenta que dicho valor es de 105 en el 85-90\% de los casos y de 135 en el resto.

- Número de WC y de urinarios: Para el modelo entreacto se han considerado entre 1 y 5 váteres y para cada uno de estos valores se ha analizado el comportamiento de los tiempos de espera cuando se tienen entre 1 y 20 urinarios. Para el modelo de flujo continuo se han considerado 102 váteres y entre 1 y 5 urinarios.

Tabla 4. Valores de las variables que intervienen en la simulación del proceso en los aseos de caballeros.

\begin{tabular}{|c|c|c|c|c|}
\hline & & & Entreacto & Flujo continuo \\
\hline \multirow{3}{*}{ Llegadas } & \multicolumn{2}{|c|}{ Número total de llegadas } & $20 ; 30 ; 40 ; 50$ & \\
\hline & \multicolumn{2}{|c|}{ Ritmo de llegadas por hora } & - & $30 ; 60 ; 90 ; 120$ \\
\hline & \multicolumn{2}{|c|}{ Rango tiempo de llegada (s) } & 5 minutos & por hora \\
\hline \multirow{6}{*}{$\begin{array}{l}\text { Tiempo de uso } \\
\text { (en segundos) }\end{array}$} & \multirow{3}{*}{ Hipótesis 1} & Tiempo uso urinario & \multicolumn{2}{|c|}{$\mathrm{N}(36 ; 5)$} \\
\hline & & Tiempo uso WC (opción urinario) & \multicolumn{2}{|c|}{$\mathrm{N}(90 ; 15)$} \\
\hline & & Tiempo uso WC (opción WC) & \multicolumn{2}{|c|}{$\mathrm{N}(90 ; 15)$} \\
\hline & \multirow{3}{*}{ Hipótesis 2} & Tiempo uso urinario & \multicolumn{2}{|c|}{$\mathrm{N}(45 ; 5)$} \\
\hline & & Tiempo uso WC (opción urinario) & \multicolumn{2}{|c|}{$\mathrm{N}(90 ; 15)$} \\
\hline & & Tiempo uso WC (opción WC) & \multicolumn{2}{|c|}{$\mathrm{N}(115 ; 20)$} \\
\hline \multicolumn{3}{|c|}{ Proporción que prefieren váter } & 0,10 & 0,15 \\
\hline \multirow{2}{*}{ Puntos de servicio } & \multicolumn{2}{|c|}{ Número de WC } & $1-5$ & $1-2$ \\
\hline & \multicolumn{2}{|c|}{ Número de urinarios } & $1-20$ & $1-5$ \\
\hline
\end{tabular}

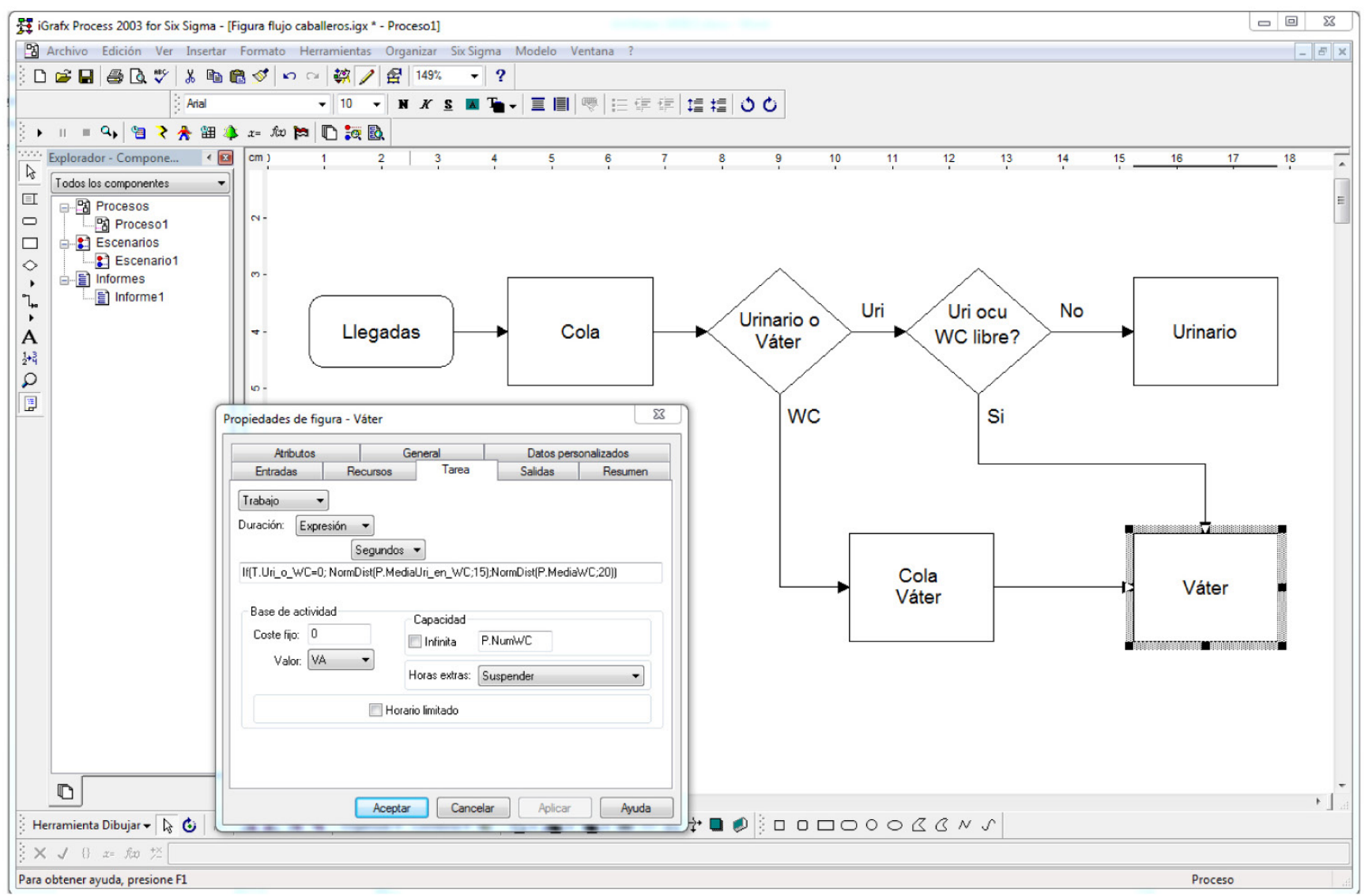

Figura 3. Pantalla de iGrafx con el diagrama de flujo correspondiente a los hombres y una ventana de diálogo donde se configura el perfil de cada una de las actividades. 


\section{SIMULACIÓN}

Hemos utilizado el programa iGrafx $®$ Process for Six Sigma (Figura 3) que permite simular procesos almacenando todas las variables asociadas a cada uno de los individuos que intervienen (características del individuo, hora de llegada, tiempo de espera, tiempo de uso...). Esto permite realizar un análisis muy detallado de lo que ha estado ocurriendo y de los factores que influyen, pudiendo elegir las variables que mejor resumen el comportamiento del proceso.

A título de ejemplo la Figura 4 representa los tiempos de espera obtenidos en una simulación del modelo entreacto para mujeres suponiendo que acuden 40, hay 8 WC y bajo la hipótesis 2 de tiempo de uso. Como se puede observar, al haber $8 \mathrm{WC}$, las 8 primeras mujeres no tienen que esperar ( 8 puntos sobre el valor cero), mientras para las otras 32 los tiempos de espera se distribuyen entre 25 y 210 segundos aproximadamente.

Para tener unos resultados más consolidados, no sujetos a las casualidades que pueden aparecer en una simulación concreta, en todos los casos se ha repetido 1.000 veces la simulación, es decir, se ha simulado lo que ocurre en 1.000 entreactos cuando se utiliza este modelo, o en 1.000 períodos

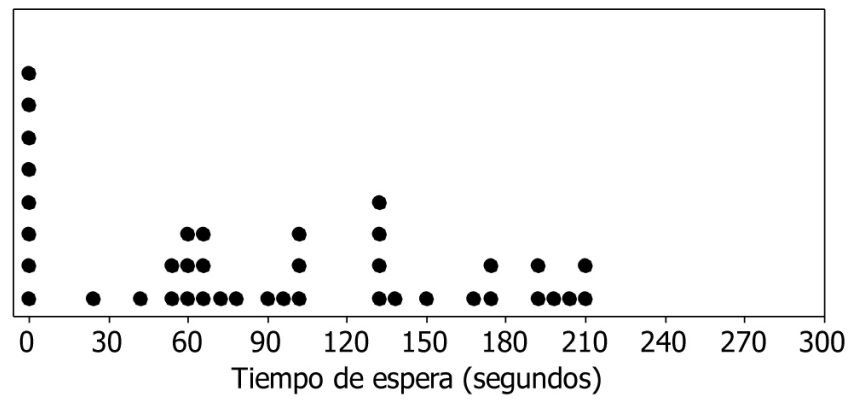

Figura 4. Tiempo de espera obtenido en una simulación del modelo entreacto para mujeres. Acuden 40 personas y hay $8 \mathrm{WC}$, con la hipótesis central de tiempo de uso. de afluencia cuando se simula un flujo continuo, acumulando todos los resultados obtenidos. Así, por ejemplo, al acumular los resultados de simular 1.000 veces la situación del ejemplo anterior se obtienen los valores representados en la Figura 5 . Las 8 personas que no tienen que esperar porque cuando llegan todavía hay WC libres ahora se convierten en algo más de 8.000 (las 8 que llegan primero cada día más alguna que, aunque llega más tarde, por casualidad también encuentra un váter vacío). Para las otras los tiempos de espera presentan la distribución que se observa en la figura.

Los tiempos de espera necesariamente presentan variabilidad y deben ser tratados en términos estadísticos. La primera idea podría ser caracterizar los tiempos de espera en cada situación a través del tiempo medio, pero sin duda éste es un valor muy poco adecuado en un caso como el nuestro, donde lo que se pretende es evitar que algunas personas deban esperar mucho tiempo, y los valores medios enmascaran estos valores con los de otras personas que apenas tienen que esperar.

Un posible valor a considerar sería el tiempo máximo de espera en cada situación, pero al repetir muchas veces la simulación pueden aparecer valores extremos debido a una confluencia de raras casualidades, por lo que ese valor no puede tomarse como referencia. Se ha optado por elegir el valor del percentil del 99\%, es decir, el valor que deja por encima sólo el $1 \%$ de los tiempos de espera registrados. De esta forma se evita que aparezcan valores anómalos y se tiene un valor que representa el tiempo máximo en la inmensa mayoría de las veces.

\section{RESULTADOS}

Para el caso de las mujeres y con el modelo entreacto la Figura 6 muestra los tiempos de espera (percentil del 99\%) en función del número de váteres disponibles y del número de personas que acuden (n). En cada gráfico se ha representado la línea obtenida con los tiempos que corresponden a la hipótesis 1 (puntos redondos) y la hipótesis 2 (puntos cuadrados, por en-

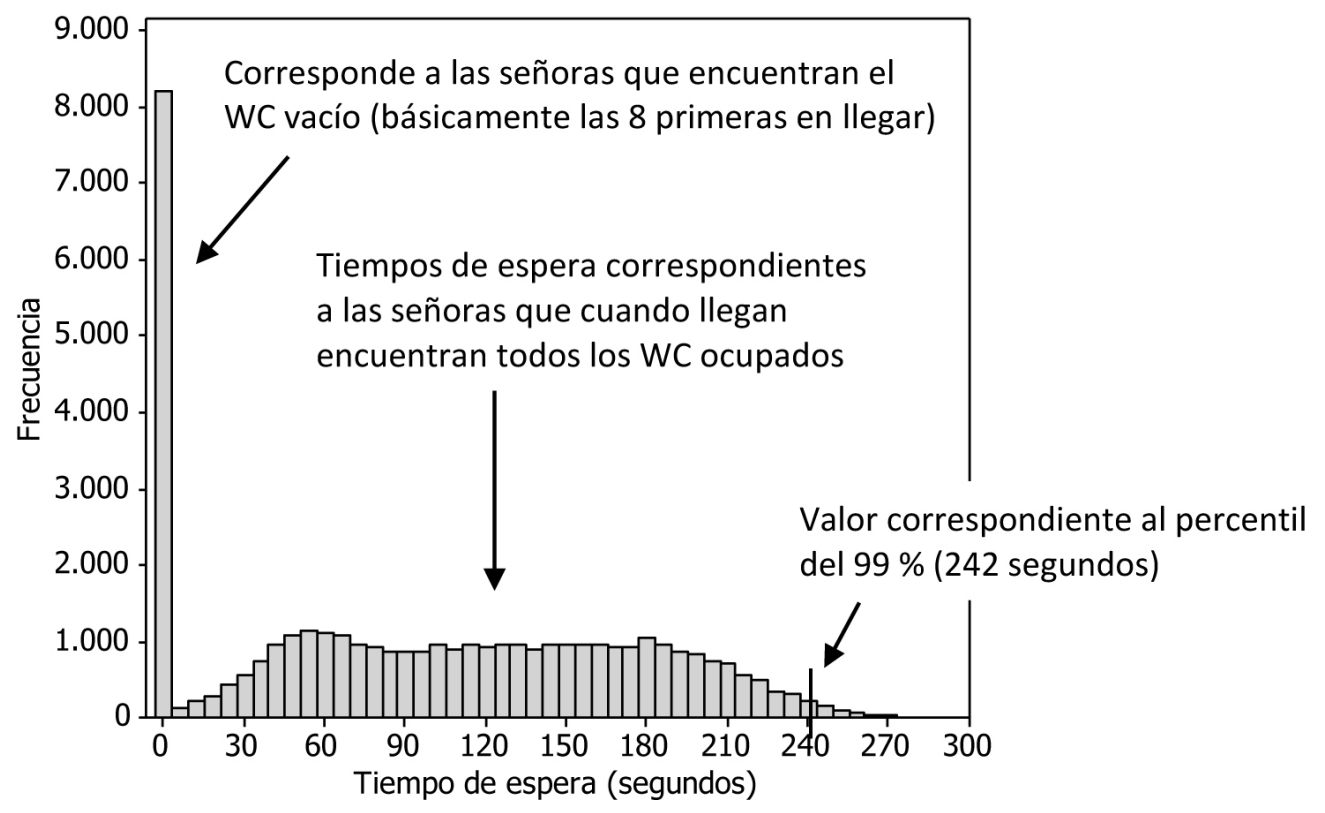

Figura 5. Tiempos de espera obtenidos al repetir 1.000 veces la simulación del modelo entreacto para mujeres (acuden 40, hay 8 WC y con la hipótesis 2 de tiempo de uso). 

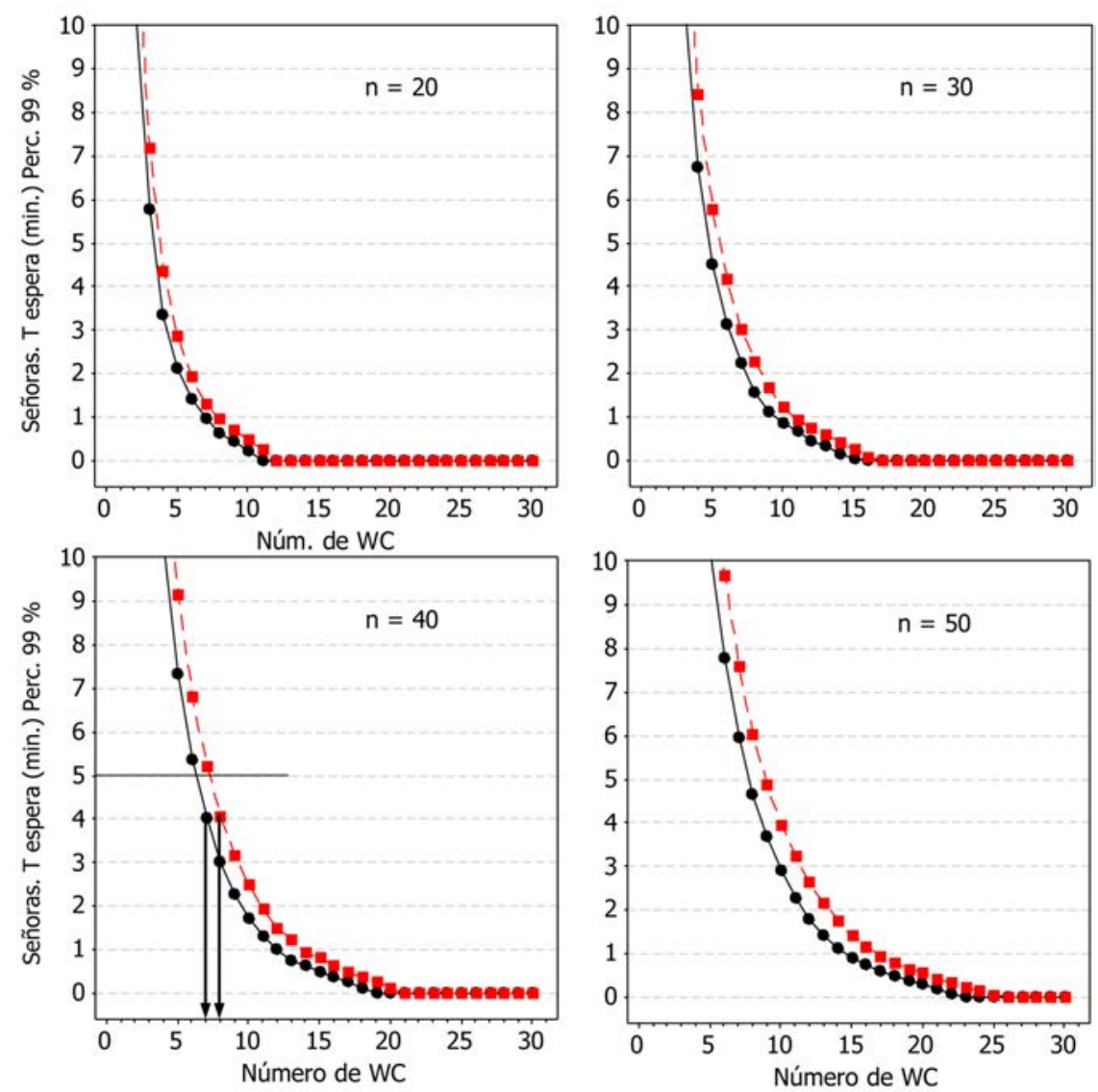

Figura 6. Tiempo de espera (percentil del 99\%, en minutos) para el caso de las mujeres con el modelo entreacto, en función del número de WC, con las hipótesis 1 (puntos redondos) y 2 (puntos cuadrados) para los tiempos de uso.

cima de la anterior). Cabe destacar que las dos líneas aparecen muy próximas y, naturalmente, en ambos casos el tiempo de espera disminuye al aumentar el número de váteres.

Si tomamos, por ejemplo, el gráfico que corresponde a una afluencia de 40 mujeres, podemos observar que para tener un tiempo de espera menor de 5 minutos habrá que colocar 90 10 váteres según sea la hipótesis considerada sobre el tiempo de uso.

Para representar los resultados para los hombres es necesario considerar tanto el número de urinarios como el de váteres. La Figura 7 muestra los tiempos de espera para el modelo entreacto cuando acuden 40 hombres y según haya entre 2 y 5 váteres y entre 1 y 20 urinarios.

Puede observarse que para tener tiempos de espera por debajo de 5 minutos basta con disponer de 2 váteres y 203 urinarios según sea hipótesis considerada sobre el tiempo de uso.

Con el modelo de flujo continuo los requerimientos de puntos de servicio son claramente menores. En el caso de las mujeres (Figura 8), suponiendo un flujo de 90 personas/hora se necesitan 4 o 5 váteres (según la hipótesis sobre tiempos de uso) para tener tiempos de espera por debajo de 2 minutos.

Para los hombres, con el mismo flujo de afluencia de 90 personas/hora y fijando en 2 el número de váteres, un solo urinario permite tiempos de espera por debajo de 3 minutos, también con independencia de la hipótesis sobre los tiempos de uso (Figura 9).
Estos resultados también se pueden visualizar a través del número de váteres (señoras) y de váteres y urinarios (caballeros) necesarios para que los tiempos de espera estén por debajo de un determinado valor. La Tabla 5 muestra los resultados para un tiempo de espera por debajo de 5 minutos con el modelo entreacto, y por debajo de 2 minutos con el de flujo continuo.

Puede parecer que los 5 minutos elegidos para el modelo entreacto son un valor exagerado, pero hay que tener en cuenta que en muy pocas personas se llegará a ese valor. En primer lugar porque se ha elegido el número de puntos de servicio que hacen que el percentil del $99 \%$ esté siempre por debajo de 5 minutos y así, en el ejemplo ya comentado de las mujeres, cuando acuden 40 y se proponen 7 u 8 váteres, con esos valores el percentil del $99 \%$ queda aproximadamente en 4 minutos, ya que elegir valores inferiores para el número de váteres lo dejaría por encima de los 5 . Por otra parte, los tiempos de espera para cada persona en un caso como este son los que se han representado en la Figura 5 y corresponden a las proporciones que se indican en la Tabla 6 , que consideramos unos valores razonables.

Para el modelo de flujo continuo, al tener unos requerimientos de espacio más reducidos, se ha optado por un tiempo máximo de 2 minutos, lo cual significa que raramente se deberá esperar. En cualquier caso, a partir de las gráficas presentadas (Figuras 7, 8, 9 y 10) se puede elegir el número de puntos de servicio que corresponda al tiempo de espera deseado.

Se observa también en estas gráficas que la hipótesis sobre los tiempos de uso afecta poco a los resultados obtenidos 

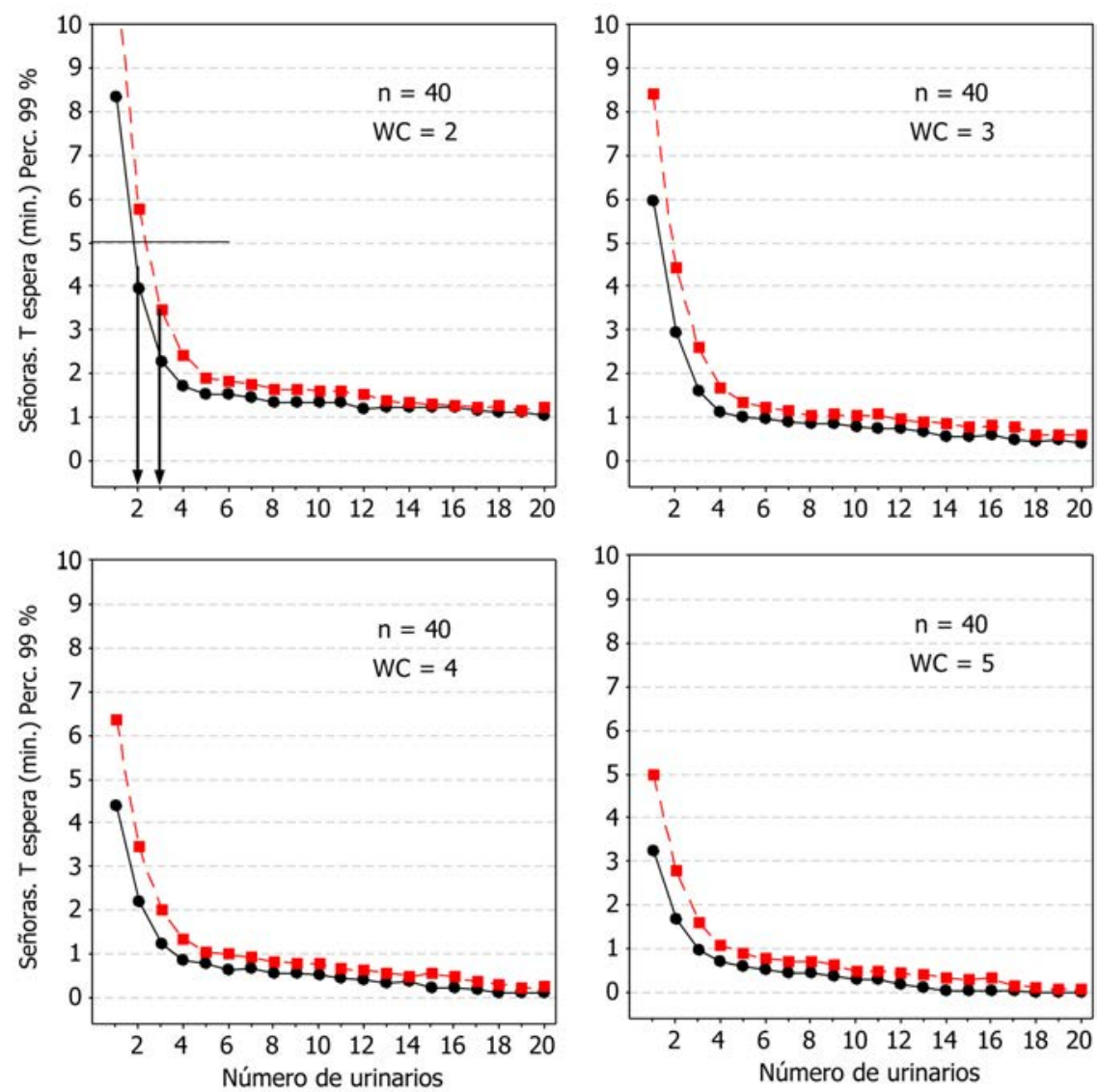

Figura 7. Tiempos de espera (percentil del 99\%, en minutos) en el caso de los hombres con el modelo entreacto, en función de número de WC y de urinarios, para una afluencia de 40 personas (los puntos redondos corresponden a la hipótesis 1 sobre el tiempo de uso y los cuadrados a la hipótesis 2).
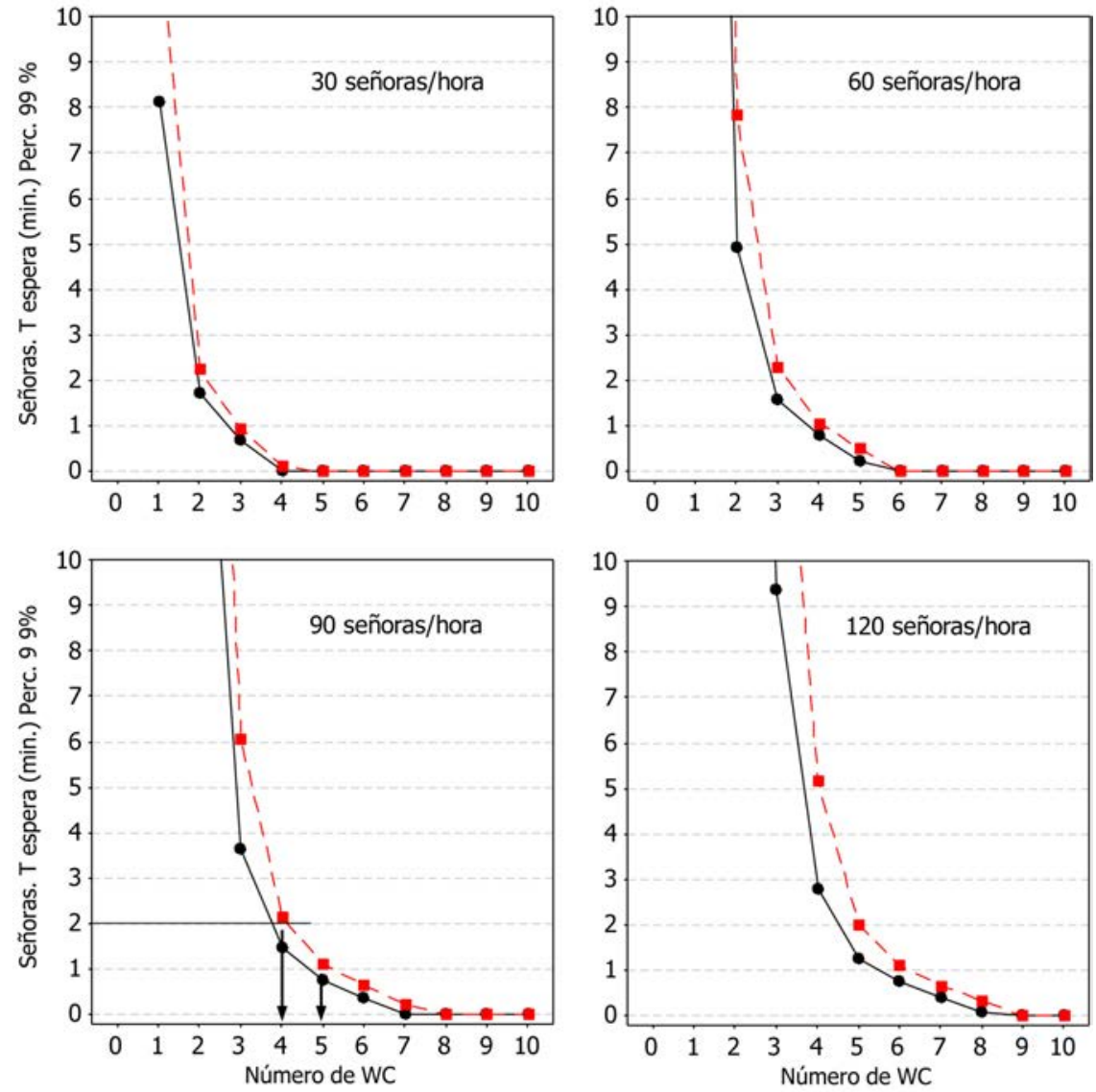

Figura 8. Tiempos de espera (en minutos, percentil del 99\%) para el modelo de flujo continuo en el caso de las mujeres con las hipótesis 1 (puntos redondos) y 2 (puntos cuadrados) para los tiempos de uso. 

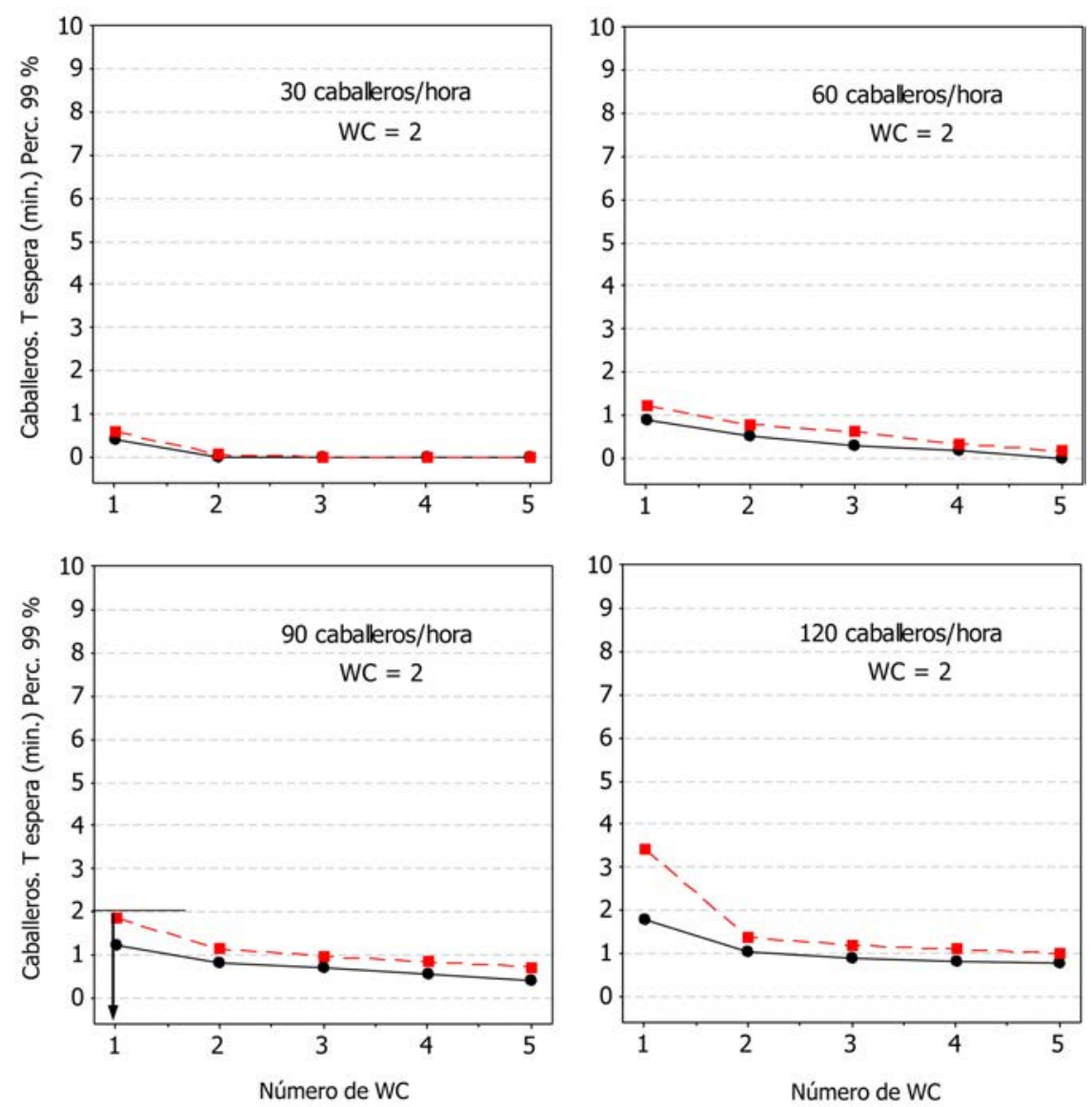

Figura 9. Tiempos de espera (en minutos, percentil del 99\%) para el modelo de flujo continuo en el caso de los hombres, con las hipótesis 1 (puntos redondos) y 2 (puntos cuadrados) para los tiempos de uso.

Tabla 5. Número de puntos de servicio necesarios para tener tiempos de espera por debajo de 5 minutos (entreacto) o de 2 minutos (flujo continuo) en más del 99\% de los casos.

\begin{tabular}{|c|c|c|c|}
\hline \multicolumn{4}{|c|}{ Modelo entreacto (T espera < 5 min.) } \\
\hline $\begin{array}{c}\text { Llegadas } \\
\text { en 5 min. }\end{array}$ & $\begin{array}{c}\text { Hipótesis } \\
\text { sobre T uso }\end{array}$ & $\begin{array}{c}\text { Señoras } \\
\text { (WC) }\end{array}$ & $\begin{array}{c}\text { Caballeros } \\
\text { (WC-urinarios) }\end{array}$ \\
\hline \multirow{2}{*}{20} & $\mathrm{H} 1$ & 4 & $1-1$ \\
\cline { 2 - 4 } & $\mathrm{H} 2$ & 4 & $1-2$ \\
\hline \multirow{2}{*}{30} & $\mathrm{H} 1$ & 5 & $1-2$ \\
\cline { 2 - 4 } & $\mathrm{H} 2$ & 6 & $1-3$ \\
\hline \multirow{2}{*}{40} & $\mathrm{H} 1$ & 7 & $2-2$ \\
\cline { 2 - 4 } & $\mathrm{H} 2$ & 8 & $2-3$ \\
\hline \multirow{2}{*}{50} & $\mathrm{H} 1$ & 8 & $2-3$ \\
\cline { 2 - 4 } & $\mathrm{H} 2$ & 9 & $2-3$ \\
\hline
\end{tabular}

\begin{tabular}{|c|c|c|c|}
\hline \multicolumn{4}{|c|}{ Modelo de flujo continuo (T espera < 2 min.) } \\
\hline $\begin{array}{c}\text { Llegadas } \\
\text { por hora }\end{array}$ & $\begin{array}{c}\text { Hipótesis } \\
\text { sobre T uso }\end{array}$ & $\begin{array}{c}\text { Señoras } \\
\text { (WC) }\end{array}$ & $\begin{array}{c}\text { Caballeros } \\
\text { (WC-urinarios) }\end{array}$ \\
\hline \multirow{2}{*}{30} & $\mathrm{H} 1$ & 2 & $1-1$ \\
\cline { 2 - 4 } & $\mathrm{H} 2$ & 3 & $1-1$ \\
\hline \multirow{2}{*}{60} & $\mathrm{H} 1$ & 3 & $1-1$ \\
\cline { 2 - 4 } & $\mathrm{H} 2$ & 4 & $1-2$ \\
\hline \multirow{2}{*}{90} & $\mathrm{H} 1$ & 4 & $2-1$ \\
\cline { 2 - 4 } & $\mathrm{H} 2$ & 5 & $2-1$ \\
\hline \multirow{2}{*}{$12 \mathrm{O}$} & $\mathrm{H} 1$ & 5 & $2-1$ \\
\cline { 2 - 4 } & $\mathrm{H} 2$ & 6 & $2-2$ \\
\hline
\end{tabular}

(como máximo un váter para las mujeres y un urinario para los hombres), por lo que podemos decir que la elección de los tiempos de uso, entre los valores considerados, no tiene una importancia crítica.

Tabla 6. Tabulación de los tiempos de espera obtenidos con modelo entreacto para mujeres cuando acuden 40, hay 8 WC y con la hipótesis 2 de tiempo de uso.

\begin{tabular}{|c|c|}
\hline Tiempo de espera (min.) & \% \\
\hline 0 & 20,4 \\
\hline $0-1$ & 15,2 \\
\hline $1-2$ & 23,7 \\
\hline $2-3$ & 23,6 \\
\hline $3-4$ & 16,1 \\
\hline$>4$ & 1,2 \\
\hline
\end{tabular}

\section{EQUILIBRADO DE LOS TIEMPOS DE ESPERA}

Los tiempos de espera dependen de la afluencia, del número de puntos de servicio y, en mayor o menor medida, del resto de variables consideradas. Sin embargo, con independencia de cuales sean estas condiciones, las simulaciones realizadas ponen de manifiesto que para lograr unos tiempos de espera similares el número de puntos de servicio para las mujeres deben ser de entre 1,5 y 2 veces el de hombres (Tabla 7). En algún caso este ratio queda fuera del intervalo recomendado, pero ello es debido a que el número de puntos de servicio es pequeño, de forma que el ratio es muy sensible a cambios en una sola unidad.

Supongamos, a título de ejemplo, que para construir los aseos en un teatro se dispone de unos 12 metros lineales y que un 
Tabla 7. Ratio del número de puntos de servicio necesarios en los aseos de señoras respecto a los de caballeros para que el tiempo de espera (percentil del 99\%) esté por debajo del valor que se indica.

\begin{tabular}{|c|c|c|}
\hline \multicolumn{3}{|c|}{ Modelo entreacto (T espera < 5 min.) } \\
\hline $\begin{array}{c}\text { Llegadas } \\
\text { en 5 min. }\end{array}$ & $\begin{array}{c}\text { Hipótesis sobre } \\
\text { T uso }\end{array}$ & $\begin{array}{c}\text { Ratio } \\
\text { señoras/caballeros }\end{array}$ \\
\hline \multirow{2}{*}{20} & $\mathrm{H} 1$ & 2,00 \\
\cline { 2 - 3 } & $\mathrm{H} 2$ & 1,33 \\
\hline \multirow{2}{*}{30} & $\mathrm{H} 1$ & 1,67 \\
\cline { 2 - 3 } & $\mathrm{H} 2$ & 1,50 \\
\hline \multirow{2}{*}{40} & $\mathrm{H} 1$ & 1,75 \\
\cline { 2 - 3 } & $\mathrm{H} 2$ & 1,60 \\
\hline \multirow{2}{*}{50} & $\mathrm{H} 1$ & 1,60 \\
\cline { 2 - 3 } & $\mathrm{H} 2$ & 1,80 \\
\hline
\end{tabular}

\begin{tabular}{|c|c|c|}
\hline \multicolumn{3}{|c|}{ Modelo flujo continuo (T espera < 2 min.) } \\
\hline $\begin{array}{c}\text { Llegadas } \\
\text { por hora }\end{array}$ & $\begin{array}{c}\text { Hipótesis sobre T } \\
\text { uso }\end{array}$ & $\begin{array}{c}\text { Ratio } \\
\text { señoras/caballeros }\end{array}$ \\
\hline \multirow{2}{*}{30} & $\mathrm{H} 1$ & 1,00 \\
\cline { 2 - 3 } & $\mathrm{H} 2$ & 1,50 \\
\hline \multirow{2}{*}{60} & $\mathrm{H} 1$ & 1,50 \\
\cline { 2 - 3 } & $\mathrm{H} 2$ & 1,33 \\
\hline \multirow{2}{*}{90} & $\mathrm{H} 1$ & 1,33 \\
\cline { 2 - 3 } & $\mathrm{H} 2$ & 1,67 \\
\hline \multirow{2}{*}{120} & $\mathrm{H} 1$ & 1,67 \\
\cline { 2 - 3 } & $\mathrm{H} 2$ & 1,50 \\
\hline
\end{tabular}

Tabla 8. Número de puntos de servicio recomendado según sea el modelo y el número de llegadas considerado.

\begin{tabular}{|c|c|c|}
\hline \multicolumn{3}{|c|}{ Entreacto. T espera < 5 min. } \\
\hline $\begin{array}{c}\text { Llegadas en 5 } \\
\text { min. }\end{array}$ & $\begin{array}{c}\text { Señoras } \\
\text { (WC) }\end{array}$ & $\begin{array}{c}\text { Caballeros } \\
\text { (WC-urinarios) }\end{array}$ \\
\hline 20 & 4 & $1-2$ \\
\hline 30 & 6 & $1-3$ \\
\hline 40 & 8 & $2-3$ \\
\hline 50 & 9 & $2-3$ \\
\hline
\end{tabular}

\begin{tabular}{|c|c|c|}
\hline \multicolumn{3}{|c|}{ Flujo continuo. T espera < 2 min. } \\
\hline $\begin{array}{c}\text { Llegadas } \\
\text { por hora }\end{array}$ & $\begin{array}{c}\text { Señoras } \\
\text { (WC) }\end{array}$ & $\begin{array}{c}\text { Caballeros } \\
\text { (WC-urinarios) }\end{array}$ \\
\hline 30 & 3 & $1-1$ \\
\hline 60 & 4 & $1-2$ \\
\hline 90 & 5 & $2-1$ \\
\hline 120 & 6 & $2-2$ \\
\hline
\end{tabular}
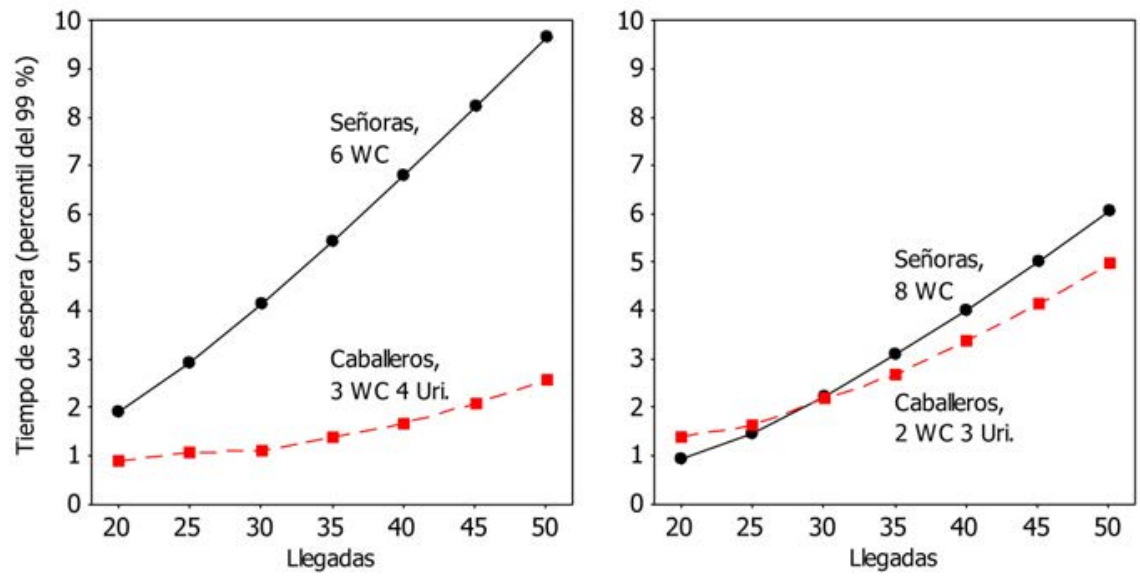

Figura 10. Tiempos de espera con el modelo entreacto y el número de puntos de servicio que se incida (con la hipótesis 2 sobre los tiempos de uso).

váter ocupa 1 metro mientras que un urinario ocupa $70 \mathrm{~cm}$. Dividir este espacio a partes iguales entre hombres y mujeres, colocando 6 váteres para las mujeres (ocupando 6 metros) y, por ejemplo, 3 váteres y 4 urinarios para los hombres (ocupando 5,8 metros) es una solución que produce una formación de colas y unos tiempos de espera muy diferentes entre unos y otros, tal como se puede ver en la Figura 10, izquierda. Sin embargo, si el reparto se realiza colocando 8 váteres en los aseos de señoras (8 metros) y 2 váteres y 3 urinarios en el de caballeros $(4,1$ metros) los tiempos de espera son similares.

\section{CONCLUSIONES. RECOMENDACIONES PARA DISTRIBUCIÓN DE ESPACIOS}

Cuando la afluencia de hombres y mujeres puede considerarse similar, las simulaciones realizadas ponen de manifiesto que la mejor manera de repartir el espacio disponible es asignando entre 1,5 y 2 veces más puntos de servicio a los aseos de señoras que a los de caballeros. De esta forma tienden a equilibrarse los tiempos de espera, evitando que las mujeres deban esperar mucho más que los hombres, como viene siendo habitual.

Respecto al número concreto de puntos de servicio que hay que colocar, la Tabla 8 presenta unas recomendaciones según se trate del modelo entreacto o de flujo continuo. Se han elegido los valores que garantizan que el percentil del $99 \%$ de los tiempos de espera estará por debajo de 5 minutos en modelo entreacto y de 2 minutos en el de flujo continuo.

Aunque hemos visto que los resultados obtenidos no dependen de forma importante de la hipótesis sobre el tiempo de uso (la basada en las indicaciones de las British Standards o la que considera unos valores medios algo superiores), hemos considerado más prudente usar los resultados con los tiempos de uso superiores. Una razón para inclinarnos por esta opción es que existen detalles como la facilidad de movimiento dentro de los aseos, así como de visión de váteres vacíos, 
o el tiempo que transcurre entre que una persona sale y otra entra que pueden enlentecer el proceso y que, de esta forma, pueden considerarse también incorporados.

Para usar estas recomendaciones sobre el número de puntos de servicio es necesario hacer también una hipótesis sobre el porcentaje de potenciales usuarios que usarán los aseos, y sobre este particular no hemos encontrado ninguna referencia concreta. Las British Standards dicen que cuando el acceso está restringido a un corto período de tiempo, como en las paradas de viajes largos, el porcentaje de personas que desean ir al aseo puede llegar al $80 \%$, pero sin duda éste es un valor muy exagerado para los intermedios de espectáculos. Cuando los aseos son accesibles todo el tiempo, cita un 5\% como número de usuarios respecto al total de visitantes. Para intermedios de espectáculos entendemos razonable considerar valores que pueden rondar el $15 \%$ del total. Nuestras re- comendaciones son muy similares a las que realiza el Código de Construcción Australiano (15) si se considera que visitan los aseos el $12 \%$ del aforo total del local.

Por último, deseamos poner de manifiesto que nuestras recomendaciones sólo pretenden evitar errores evidentes en el diseño de los aseos públicos, esos que producen largas colas en los aseos de señoras mientras que en los de caballeros se circula con total fluidez. Para mejorar estas recomendaciones se debería estudiar sobre el terreno cuales son los tiempos de uso, la proporción de personas que van a los aseos en el intermedio de los espectáculos y los períodos en que se produce la máxima afluencia. Nunca se podrá predecir exactamente lo que va a ocurrir porque nos movemos en un terreno de incertidumbre y variabilidad, pero, sin duda, se podrán dar unas recomendaciones que resulten adecuadas en la inmensa mayoría de los casos.

\section{REFERENCIAS}

(1) Ibáñez, M. E. (1999). La ópera vuelve a BCN. Las reacciones. El Periódico de Catalunya, 8-10-1999 (p. 4).

(2) Cortes Generales (1982). Real Decreto 2816/1982: Reglamento General de Policía de Espectáculos Públicos y Actividades Recreativas. Boletín Oficial del Estado, n. ${ }^{\circ} 267$. España.

(3) Greed, C. (2003). Inclusive Urban Design. Public Toilets, Oxford: Architectural Press.

(4) Scott Brown, D. (1967). Planning the Powder Room. Journal of the American Institute of Architects, April 1967, pp. 8183 (traducido y reeditado en 2013 en Armada de Palabras, pp. 150-157, México DF: Arquine).

(5) Edwards, J., McKie, L. (1996). A Serious Issue for the Body Politic. European Journal of Women's Studies, 3(3): 215230, doi: http://dx.doi.org/10.1177/135050689600300303.

(6) Gaffin, G. (1994). So What? P's and Q's. Paediatric and Perinatal Epidemiology, 8(1): 25-26, doi: http://dx.doi. org/10.1111/j.1365-3016.1994.tboo432.x.

(7) García Nieto, J., de la Peña, E. (2001). El cuarto de baño en la vivienda urbana, Madrid: Fundación Cultural COAM.

(8) García Nieto, J., de la Peña, E. (2001). El diseño de la higiene. Origen y evolución histórica de los aparatos sanitarios, Madrid: Fundación Cultural COAM.

(9) Lupton, E., Miller, J. A. (1992). The Bathroom, the Kitchen, and the Aesthetics of Waste: A Process of Elimination, Cambridge (USA): MIT List Visual Arts Center.

(10) Kira, A. (1977). The Bathroom, New York: Bantam Books.

(11) García Nieto, J., de la Peña, E. (2002). Manual de equipamiento higiénico de los edificios. Legislación estatal y autonómica y recomendaciones de diseño, Madrid: Dilex, S. L.

(12) International Organization for Standardization (ISO). http://www.iso.org/iso/home/store/catalogue_ics.htm

(13) Cortes Generales (2010). Orden VIV/561/2010: Documento técnico de condiciones básicas de accesibilidad y no discriminación para el acceso y utilización de los espacios públicos urbanizados. Boletín Oficial del Estado, n. ${ }^{\circ} 61$. España.

(14) British Standards Institution (2006). BS 6465-1:2006 Sanitary Installations.

(15) Australian Building Codes Board (2015). National Construction Code 2015, vol. 1, part F2 «Sanitary and Other Facilities». http://services.abcb.gov.au/NCCOnline/Publications/2015/Volume1\#TableF2.3 (el acceso es gratuito, pero necesita registro previo).

(16) Peña, D. (2008). Fundamentos de Estadística, Madrid: Alianza Editorial. 